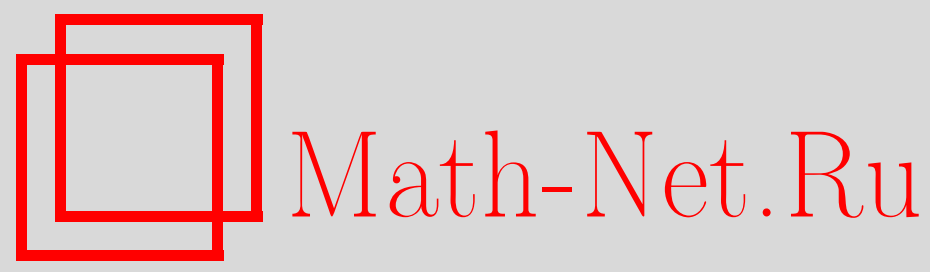

М. Ф. Насрутдинов, Полулокальные групповые алгебры, Матем. заметки, 2005, том 78, выпуск 3, 409-412

DOI: https://doi.org/10.4213/mzm2597

Использование Общероссийского математического портала Math-Net.Ru подразумевает, что вы прочитали и согласны с пользовательским соглашением http://www.mathnet.ru/rus/agreement

Параметры загрузки:

IP: 54.196 .121 .252

26 апреля 2023 г., 05:09:15

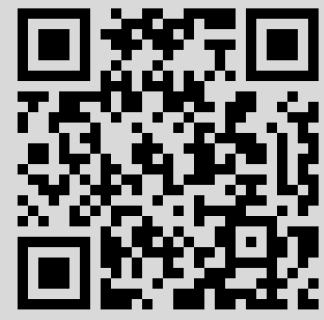




\section{ПОЛУЛОКАЛЬНЫЕ ГРУППОВЫЕ АЛГЕБРЫ}

\section{М. Ф. Насрутдинов}

Пусть $k[G]$ - полулокальная групповая алгебра. Показано, что если $k$ - алгебраически замкнутое поле, то всякий конечно порожденный плоский $k[G]$-модуль проективен.

Библиографоия: 14 названий.

Рассматриваются ассоциативные кольца с единицей и правые унитарные модули над ними. Мы будем придерживаться обозначений и терминологии двухтомника Фейса [1] для теории колец и модулей, а также обзора [2] и книги [3] для теории групповых колец.

Пусть $R$ - кольцо. Будем обозначать через $J(R)$ радикал Джекобсона кольца $R$. Кольцо $R$ назьвается полулокальным (локальным, квазилокальным.), если его факторкольцо $R / J(R)$ удовлетворяет условию обрыва убывающих цепей правых идеалов (является телом, простьм артиновьп кольцом, соответственно). В классической работе Басса [2] (см. также [1, т. 2]) было показано, что всякий плоский правый $R$-модуль проективен тогда и только тогда, когда $R$ - полулокальное кольцо и его радикал Джекобсона $T$-нильпотентен слева, т.е. для всякой последовательности элементов $a_{1}, \ldots$, $a_{n}, \ldots$ из $J(R)$ существует $k$ такое, что $a_{k} a_{k-1} \cdots a_{1}=0$. Такие кольца назьваются $c o$ вериенны.ми (справа). Естественно поставить вопрос о строении колец над которьми проективны все конечно порожденные плоские модули. К классу таких колец принадлежат полусовершенные кольца, квазилокальныекольца [5] и кольца, вложимые в тела [6]. Проективности конечно порожденных плоских модулей посвящены, в частности, работы $[5]-[9]$.

В работе [10] (см. также [2]) показано, что групповая алгебра $k[G]$ групшы $G$ над полем $k$ совершенное кольцо тогда и только тогда, когда $G$ - конечная группа.

Мы рассматриваем вопрос при каких условиях проективен всякий конечно порожденньй плоский модуль над полулокальными групповыми алгебрами.

Известно (см., например, [11]), что в случае поля нулевой характеристики групповая алгебра $k[G]$ полулокальна тогда и только тогда, когда групша $G$ конечна. Для полей ненулевой характеристики вопрос о строении групшы остается открытым. Так, например, полулокальна групповая алгебра $k[G]$ над полем характеристики $p$, где $G$ - бесконечная конечно порожденная периодическая $p$-группа, построенная Е. С. Голодом, на основе теоремы Голода-Шафаревича.

Строение полулокальных групшовых алгебр до конца не изучено. В работе [10] было показано, что если $R[G]$ - полулокальное групповое кольцо групшы $G$ над кольцом $R$, то 
группа $G$ периодическая (см. также [12], где теорема доказана для полугрупповых колец). При переходе к подкольцам полулокальность, вообще говоря, не сохраняется. Для этого достаточно рассмотреть поле рациональных чисел и кольцо целых чисел. Однако в работе [13] Окнинский доказал, что если $R[G]$ - полулокальное групповое кольцо группы $G$ над кольцом $R$, то для любой подгруппы $H$ группы $G$ групповое кольцо $R[H]$ полулокально.

Если $H$ - подгруппа групшы $G$, то через $\omega(H) k[G]$ обозначают правьг̆ идеал $\sum_{h \in H}(1-h) k[G]$ группового кольца $k[G]$. Если $H$ - нормальная подгруппа, то $\omega(H) k[G]$ является ядром кольцевого гомоморфизма $k[G] \rightarrow k[G / H]$.

В выше приведенном примере с группой Голода фундаментальный идеал $\omega(G) k[G]$ совпадает с радикалом Джекобсона. То есть эта групповая алгебра является локальным кольцом.

Нами показано, что в случае алгебраически замкнутых полей полулокальная групповая алгебра $k[G]$ "локально" устроена подобным образом, т.е. в любой конечно порожденной подгрупе $G_{1}$ группы $G$ существует подгруппа $H$ конечного индекса такая, что $\omega(H) k\left[G_{1}\right]$ содержится в радикале Джекобсона кольца $k\left[G_{1}\right]$.

Как следствие получено, что в случае алгебраически замкнутых полей всякий конечно порожденньй плоский модуль над полулокальной групповой алгеброй проективен.

Перейдем теперь к изложению результатов статьи.

Пусть $k[G]$ - групповая алгебра. Рассмотрим групу (относительно умножения) $\widetilde{G}=$ $\{g+J(k[G]) \mid g \in G\}$ и гомоморфизм групп $\theta: G \rightarrow \widetilde{G}$. Ясно, что $\theta$ - эпиморфизм. Обозначим через $H=\operatorname{Ker}(\theta)$ нормальную подгруппу групшы $G$. Элемент $g \in H$ тогда и только тогда, когда $1-g \in J(k[G])$. Заметим, что $\omega(H) k[G] \subseteq J(k[G])$.

ТЕОРемА 1. Пусть $k[G]$ - полулокальная групповая алгебра группы $G$ над полем $k$.

1. Если характеристика поля $k$ равна $p$, то $H$ является $p$-группой.

2. В случае поля нулевой характеристики группы $G$ и $\widetilde{G}$ изоморфны.

ДокАЗАТЕЛЬСТво. Пусть $g \in H$; тогда $1-g \in J(k[G])$. Для любой подгрупшы $H<G$ имеет место включение $k[H] \cap J(k[G]) \subseteq J(k[H])$ [2, лемма 6.3]. Поэтому, $1-g \in J(k[\langle g\rangle])$, где $\langle g\rangle$ - циклическая подгрупша, порожденная $g$. Так как радикал Джекобсона групповой алгебры $k[\langle g\rangle]$ нильпотентен, то фундаментальньй идеал $\omega(k[\langle g\rangle])=J(k[\langle g\rangle])$ нильпотентен и поэтому [3, теорема 2.8] порядок элемента $g$ есть некоторая степень $p$, где $p=\operatorname{char} k$. В случае $\operatorname{char} k=0 k[\langle g\rangle]$ - полупростая алгебра и $J(k[\langle g\rangle]=0$. Доказательство окончено.

Пусть теперь $R=k[G]$ - групповая алгебра над алгебраически замкнутым полем характеристики $p$ и $G$ - конечно порожденная група. Рассмотрим кольцо $k[G / H] \cong$ $k[G] / \omega(H) k[G]$. Как эпиморфный образ полулокального кольца оно полулокально и $\omega(H) k[G] \subseteq J(k[G])$. Если $1-g H \in J(k[G / H])=J(k[G]) / \omega(H) k[G]$, то $1-g \in J(k[G])$. Значит, для групшы $G / H$ и групповой алгебры $k[G / H]$ отображение $\theta$ изоморфизм.

Отображение $\theta$ вкладывает $G / H$ в линейную группу (в произведение линейных групп). По теореме Шура (см., например, [14, теорема 52.1.3]) о локальной конечности периодической линейной группы получим конечность групшы $G / H$. Итак, справедлива 
ТЕОрема 2. Если $k[G]$ - полулокальная групповая алгебра конечно порожденной группь $G$ над алгебраически замкнутым полем $k$ характеристики $p$, то в группе $G$ существует нормальная p-подгруппа $H$ конечного индекса такая, что $\omega(H) k[G] \subseteq J(k[G])$.

Следуя работе [7], кольцо $R$ будем назьвать (правым) FGFP-кольцом, если проективен любой конечно порожденньй плоский $R$-модуль. Свойство кольца быть FGFP-кольцом наследуется подкольцами. При некоторых предположениях можно поднять свойство быть FGFP-кольцом с подкольца на все кольцо. Справедливо следующее

ПРЕДЛОЖЕНИЕ 1 [7, предложение 1.2]. Пусть $S$ u $R$-кольиа $u \varphi: S \rightarrow R$ кольиевой гомоморфизм, сохраняющий единииу и кольцо $R$, рассматриваемое как правый $S$-модуль, является конечно порожденным плоским $S$-модулем. Тогда если $S$ является FGFP-кольиом, то и $R$ является FGFP-кольцом.

ТЕОРемА 3. Если $k[G]$ - полулокальная групповая алгебра группы $G$ над алгебра-

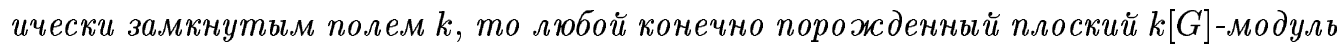
проективен.

ДокАЗАТЕльство. Докажем сначала теорему для конечно порожденных групп. Если $H$ - подгруппа конечного индекса, то $k[G]$ - свободньй, конечно порожденный $k[H]$ модуль. Поэтому по предложению 1, сформулированному выше, достаточно доказать, что $k[H]$ является FGFP-кольцом.

Рассмотрим группу $H$ теоремы 2 . Так как $\omega(H) k[H]=J(k[H])$, то $k[H]-$ локальное кольцо. Локальные кольца являются FGFP-кольцами (см., например, [5]) и теорема в этом случае доказана.

Согласно теореме 3 и следствия 4 работы [8] всякий $n$-порожденньй плоский модуль над полулокальным кольцом $R$ проективен тогда и только тогда, когда для любых матриц $r, \varepsilon \in \mathbb{R}_{n}$ таких, что $r^{2}=\varepsilon r$, вьполняется равенство $r \varepsilon^{-1} r=r$.

Пусть $r, \varepsilon$ - матрицы порядка $n$ над кольцом $k[G]$ такие, что $r^{2}=\varepsilon r$. Рассмотрим подгруппу $G_{1}$, порожденную носителями всех элементов матриц $r, \varepsilon$. Групповая алгебра $k\left[G_{1}\right]$ - полулокальная групповая алгебра конечно порожденной группы $G_{1}$, для которой теорема справедлива. Поэтому в $k\left[G_{1}\right]$, а значит и в $k[G]$, выполняется равенство $r \varepsilon^{-1} r=r$ и теорема доказана.

\section{СПИСОК ЦИТИРОВАННОЙ ЛИТЕРАТУРЫ}

[1] Фейс К. Алгебра: кольца, модули и категории. Т. 1, 2. М.: Мир, 1977, 1979.

[2] Залесский А. Е., Михалев А. В. Групповые кольца // Итоги науки и техники. Современные проблемы математики. Т. 2. М.: ВИНИТИ, 1973.

[3] Бовди А. А. Групповые кольца. Киев: УМК ВО, 1988.

[4] Bass H. Finitistic dimension and a homological generalization of semi-primary rings // Trans. Amer. Math. Soc. 1960. V. 95. P. 466-488.

[5] Сахаев И. И., Чирков Ч. В. Проективность конечно порожденных плоских модулей над полулокальным кольцом с единственным примитивным идеалом // Изв. вузов. Матем. 1970. №6. C. 100.

[6] Puninski G., Rothmaler Ph. When every finitely generated flat modules projective // J. Algebra. 2004. V. 277. P. 542-558.

[7] Jondrup S. On finitely generated flat modules // Math. Scand. 1970. V. 26. P. 233-240. 
[8] Сахаев И. И. О группе $K_{0}(A П)$ для полулокальных колец // Math. Nachr. 1987. V. 130. P. $157-175$.

[9] Сахаев И. И. О проективности конечно порожденных плоских модулей над полулокальными кольцами // Матем. заметки. 1985. Т. 37. № 2. С. 152-161.

[10] Woods S. M. On perfect group rings // Proc. Amer. Math. Soc. 1971. V. 27. № 1. P. 49-52.

[11] Okninski J. Spectrally finite and semilocal group rings // Commun. Algebra. 1980. V. 8. P. 533-541.

[12] ЖКучин А. В. О полулокальных полугрупповых кольцах // Фундамент. и прикл. матем. 1999. T. 5. №1. C. 139-147.

[13] Okninski J. Semilocal skew group rings // Proc. Amer. Math. Soc. 1980. V. 80. № 4. P. 552-554.

[14] Мерзляков Ю. И. Рациональные группы. М.: Наука, 1980.

Казанский государственньй университет

Поступило

E-mail : marat.nasrutdinov@ksu.ru

23.07.2004

Исправленный вариант

31.03 .2005 